\title{
Akute Blutung aus der linken A. transversa colli: Eine seltene Manifestation des „Sicherheitsgurt-Syndroms“
}

\section{Einleitung}

Die positiven Effekte von Sicherheitsgurten für Fahrzeuginsassen in Kraftfahrzeugen, Flugzeugen und anderen Verkehrsmitteln sind in vielen Studien belegt [exemplarisch Hodson-Walker N]. Can Med Assoc J 1970; 102: 391-393]. Dies führte zur Einführung der Gurtpflicht. Aber schon vorher wurden Gurtsysteme in Automobilen montiert (u. a.1958 Patentanmeldung des durch den Schweden Nils Bohlin entwickelten 3-Punktsicherheitsgurt durch den Autohersteller Volvo), sodass ab den 1950er-Jahren erste Berichte über Verletzungsfolgen durch Sicherheitsgurte in der Literatur erschienen. Im Jahr 1962 haben Garrett und Braunstein den Begriff „seatbelt syndrom“ geprägt [Garrett ] et al. J. Trauma 1962; 2: 220238].

Hierbei handelt es sich um eine Kombination von Verletzungen, hervorgerufen durch Kompression sowie Zug- und Scherkräfte des Gurtes auf die entsprechenden Körperregionen beim Abbremsvorgang. Beim klassischen Sicherheitsgurt-Syndrom findet man typischerweise neben der kutanen Prellmarke im Gurtverlauf Verletzungen der Wirbelsäule, des Thorax und der Abdominalorgane. Es sind jedoch weitere Verletzungen in anderen Körperregionen, z. B. der Halsorgane, möglich und beschrieben.

\section{Fallbericht}

Wir präsentieren den Fall eines 63 Jahre alten Mannes, der als Unfallopfer einer frontalen Fahrzeugkollision in das hiesige Traumazentrum gebracht wurde. Er war als Fahrer des Fahrzeugs mittels 3-Punktgurt im Fahrzeug angeschnallt. Der Patient klagte initial über Thoraxschmerzen und hatte eine Prellmarke rechts unterhalb des Rippenbogens sowie eine schmerzhafte Schwellung und Gurtmarke supraklavikulär links. Die CT-Untersuchung („Polytrauma-Spirale“) im Rahmen des üblichen Trauma-Managements zeigte eine akute Einblutung in die Halsweichteile links mit großem angrenzenden Hämatom ( Abb.1). Als Blutungsquelle wurde ein
Ast der A. subclavia links vermutet. Zudem wurde eine Sternumfraktur diagnostiziert ( Abb. 1).

Bei aktiver Blutung mit sichtbarem Kontrastmittelparavasat, klinisch rasch wachsendem Hämatom und befürchteter hämodynamischer Instabilität sowie drohenden Kompressionsfolgen durch das Hämatom (Nerven- und Organkompressionen bei weiterer Größenprogredienz) wurde eine sofortige endovaskuläre Versorgung veranlasst. Der Eingriff wurde in Lokalanästhesie und Analgosedierung über einen transfemoralen Zugang durchgeführt. Die Vitalparameter wurden durch ein Anästhesie-Team kontinuierlich überwacht.

Nach Sondierung der A. subclavia links mittels Sidewinder-II-Katheter und einem weichen, hydrophil beschichteten Draht mit gebogener Spitze wurde nach Einwechseln eines stabilen Drahtes mit Drahtspitze in der Arteria axillaris eine $80 \mathrm{~cm}$ lange $6 \mathrm{~F}-\mathrm{Schleuse}$ bis in die proximale A. subclavia links eingebracht. Zur Stabilisierung der Schleuse wurde ein relativ rigider Draht (0.018 Inch) als „buddy wire“ mit Draht- 


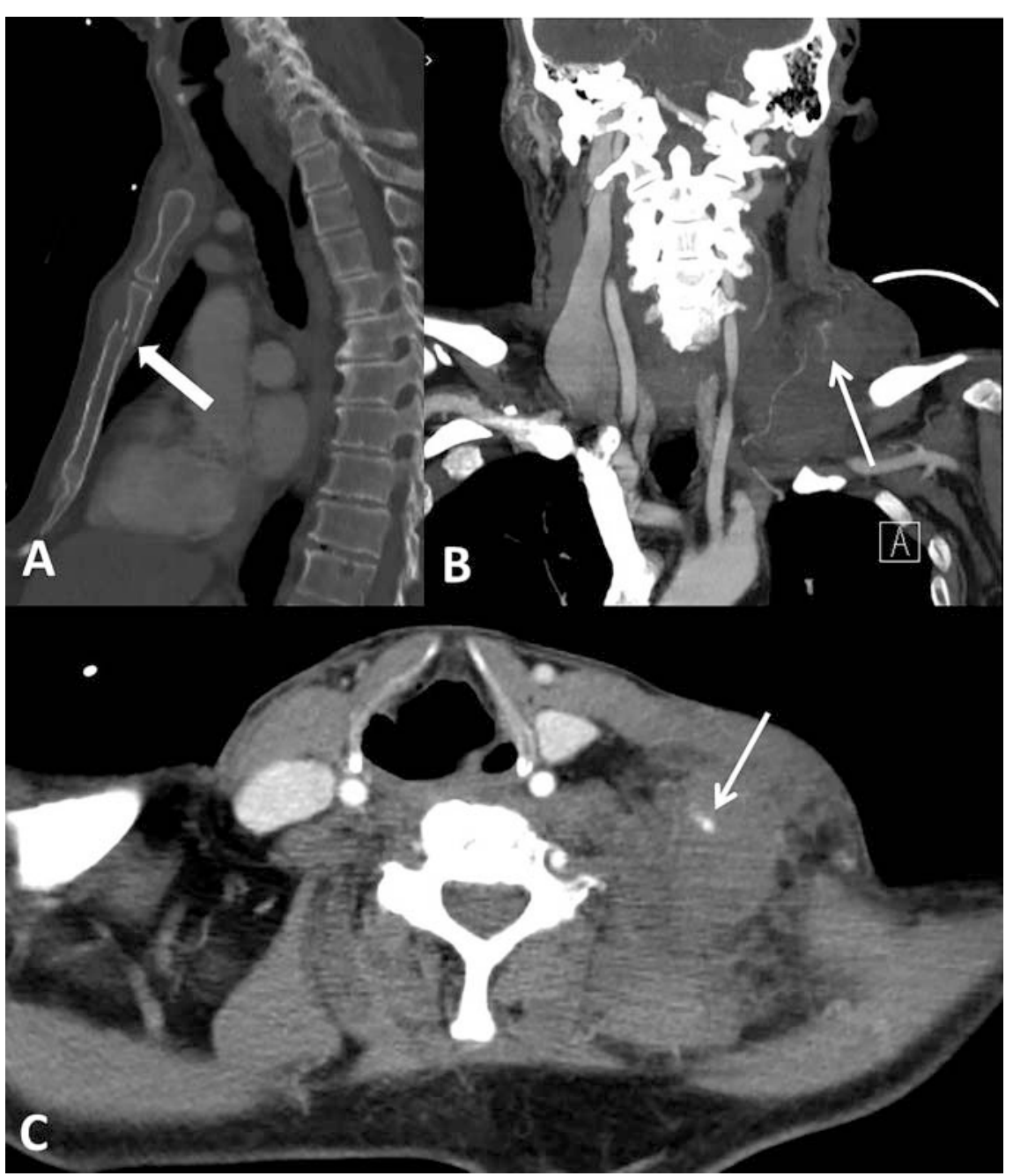

- Abb.1 CT-Untersuchung im Rahmen des Polytrauma-Managements. A Nachweis einer Sternumfraktur (dicker Pfeil). Als Korrelat der Halsschwellung findet man in der koronaren Rekonstruktion (MIP, B) und im axialen CT (C) ein großes Hämatom in den Halsweichteilen links direkt supraklavikulär mit aktiver Einblutung (Kontrastmittelparavasat siehe weiße Pfeile). Als Blutungsquelle wird ein Seitenast der A. subclavia links vermutet.

spitze in der A. axillaris links eingewechselt und verankert. Nach Gefäßdarstellung über die Schleuse ( $\triangleright$ Abb. 2) konnte die A. transversa colli als Blutungsquelle identifiziert werden. Da bei akuter Blutung auf eine Heparin-Gabe bewusst verzichtet wurde, wurde während der weiteren Intervention zur Vermeidung von Thrombenbildung innerhalb der Schleuse (mit Gefahr der Embolisation in die Arteria vertebralis links) eine Druckspülung der Schleuse durchgeführt. Die Arteria transversa colli wurde mittels Mikrokatheter und -draht zunächst in Teleskoptechnik mit einem Vertebraliskatheter als Führungskatheter selektiv sondiert und der Ramus superficialis und profundus mittels Mikrocoils verschlossen. Wegen der Nähe zu den hirnversorgenden
Arterien wurden Coils verwendet, keinerlei Partikel oder Kleber. Die Abschlussangiografie ergab ein Sistieren der Blutung ( $\triangleright$ Abb. 3).

Im stationären Verlauf konnte bei Heiserkeit als weitere zervikale Traumafolge ein vom Nasenrachenraum bis zum Hypopharynx reichendes Schleimhauthämatom, das keinerlei Therapie bedurfte, diagnostiziert werden. Nach 7 Tagen konnte der Patient bei Wohlbefinden aus dem Krankenhaus entlassen werden.

\section{Diskussion}

Die Wirksamkeit von Sicherheitsgurten bei Autounfällen beruht zum einen darauf, dass die Insassen durch die Gurte nicht unkontrolliert durch das Auto oder aus dem Auto heraus geschleudert werden können, zum anderen dehnen sich die Gurte, sodass es zu einer Verzögerung der Kraftübertragung von der Karosserie auf den menschlichen Körper kommt. Man nimmt vor allem an, dass beim Verrutschen der Gurte oder bei unsachgemäßer Anwendung gurtbedingte Verletzungen auftreten können [Schmitt $\mathrm{K}$ et al. Trauma-Biomechanik. 1. Aufl. Berlin/Heidelberg: Springer 2010].

So ist der sog. „U-Boot-Effekt“ (engl. „submarining“) beschrieben [Appel W et al. Monatsschr. Unfallheilk 1975; 78: 460-468]: Durch das schlecht fixierte Becken gleitet der Körper unter dem Gurt hindurch, wobei es zur Darmperforation oder zur Zerreißung des Mesenteriums kommen kann. Eine Perforation im Rahmen des Sicherheitsgurt-Syndroms kann sich erst klinisch nach Tagen bemerkbar machen. Ebenfalls kann der U-Boot-Effekt dazu führen, dass beim Verrutschen des Körpers der Kopf im Schultergurt hängen bleibt und der Hals stranguliert wird. Verletzungen der Halsweichteile, des Thorax und der Wirbelsäule im Rahmen des Sicherheitsgurt-Syndroms sind in der Literatur beschrieben. So wird als zervikale Verletzungsfolge relativ häufig über Larynxverletzungen, Wirbelsäulenfrakturen und Gefäßdissektionen berichtet [Hayes C et al. Radiographics 1991; 11: 23-36]. Jedoch stellt der hier präsentierte Fall die erstmalige Beschreibung einer aktiven Blutung bei Gefäßlazeration der A. transversa colli durch den Schultergurt im Rahmen eines Sicherheitsgurt-Syndroms dar.

Wichtig in der Diagnostik derartiger zervikaler Verletzungen ist, dass bei jeder erkennbaren Traumatisierung der KopfHalsregion eine kontrastmittelgestützte Darstellung der Halsarterien und Halsweichteile routinemäßig erfolgen sollte, um mögliche Traumafolgen wie Dissektionen oder Lazerationen nicht zu übersehen [AWMF-Leitlinie: Polytrauma/Schwerverletztenbehandlung, Registernummer 012-019, gültig bis 30.06.2021].

Die zeitnahe endovaskuläre Versorgung der akuten Blutung mittel Coil-Embolisation stellte in dem vorgestellten Fall eine schnelle, effektive und elegante Therapiemöglichkeit dar, wodurch eine operative 


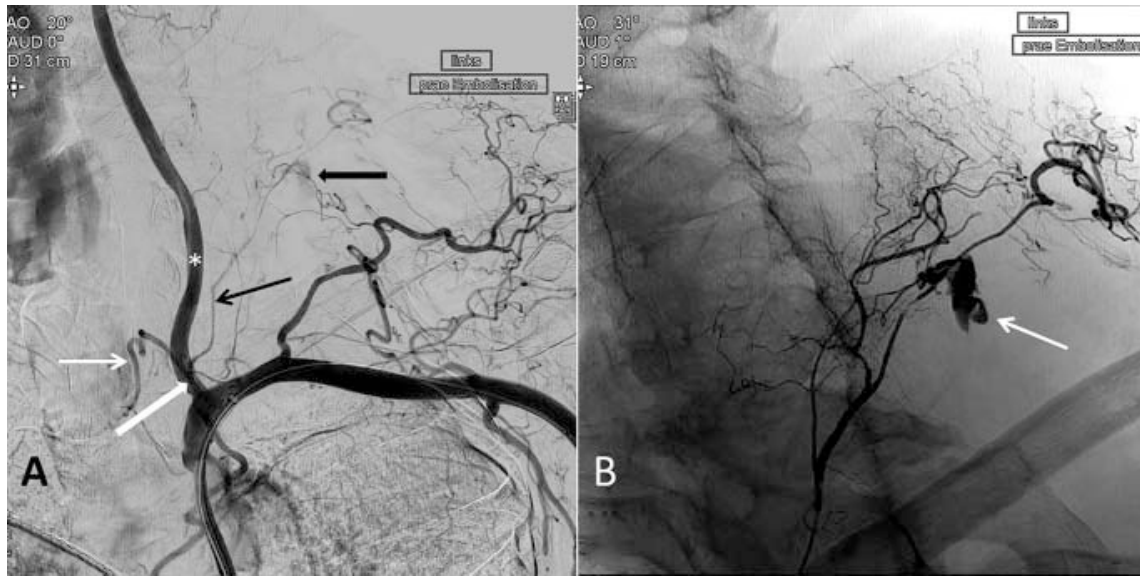

- Abb.2 A In der digitalen Subtraktionsangiografie mit Darstellung der Gefäßäste der A. subclavia links erkennt man ein flaues Kontrastmittelparavasat (schwarzer dicker Pfeil). Als Blutungsquelle wird die A. transversa colli (dünner schwarzer Pfeil), ein Ast aus dem Truncus thyrocervicalis (dicker weißer Pfeil), identifiziert. Ein weiterer Gefäßast des Truncus thyrocervicalis ist die A. thyroidea inferior (dünner weißer Pfeil). Man beachte die enge Lagebeziehung zur A. vertebralis links $\left({ }^{*}\right.$ ). B Nach selektiver Sondierung der A. transversa colli mittels Mikrokatheter und Durchführung einer digitalen Subtraktionsangiografie über den Mikrokatheter ist das Kontrastmittelparavasat in der Kontrastmittelserie deutlicher zu erkennen (weißer Pfeil).

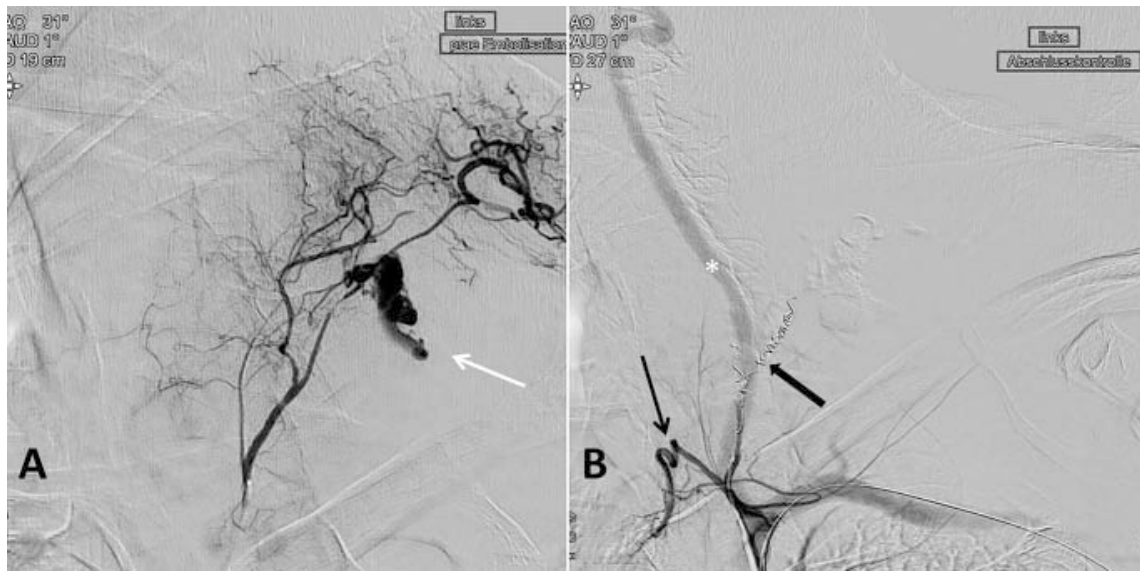

- Abb. 3 A Die A. transversa colli ist im Rahmen der akuten Blutung mit Kontrastmittelparavasat zum Teil spastisch. B Sowohl der oberflächliche als auch der tiefe Ast der A. transversa colli werden mit Microcoils verschlossen (dicker schwarzer Pfeil). In der kontrastmittelgestützten Abschlussserie erkennt man ein Sistieren der Blutung bei regelrechter Kontrastierung der A. thyroidea inferior (dünner schwarzer Pfeil), der A. subclavia und der A. vertebralis links $\left({ }^{*}\right)$.

Exploration und Ligatur des Gefäßes vermieden werden konnte.

\section{Schlussfolgerung}

Sicherheitsgurte haben einen wesentlichen Anteil bei der Vermeidung schwerer Verlet- und abhängig vom betroffenen Organ. Unter Umständen ist die Symptomatik deutlich zeitverzögert. Eine sichtbare kutane Gurtmarke sollte in der körperlichen Untersuchung auf die Problematik hindeuten. Bei Verdacht auf eine Verletzung der Halsweichteile sollte unbedingt eine kontrastmittelgestützte Darstellung der Halsarterien und Halsweichteile im Rahmen der Trauma-Bildgebung erfolgen. Der hier präsentierte Fall einer Arterienlazeration der A. transversa colli durch den Schultergurt bei angelegtem 3-Punktgurt konnte schnell und effektiv mittels endovaskulärer CoilEmbolisation behandelt werden.

Interessenkonflikt

Die Autorinnen/Autoren geben an, dass kein Interessenkonflikt besteht.

\section{Autorinnen/Autoren}

Mareike Franke ${ }^{1}$, Jörg Franke ${ }^{2}$, Kersten Mückner ${ }^{1}$

\section{${ }^{1}$ Diagnostic and Interventional Radiology, Clinic Dr. Hancken GmbH, Stade, Germany \\ ${ }^{2}$ Clinic for Trauma Surgery and Orthopedics, Elbe-Kliniken Stade-Buxtehude $\mathrm{GmbH}$, Stade, Germany}

Korrespondenzadresse

\section{Dr. Mareike Franke}

Diagnostische und Interventionelle

Radiologie, Klinik Dr. Hancken GmbH

Harsefelder Straße 8

21680 Stade

Germany

Tel.: ++49/4141/971551

mfr@hancken.de

\section{Bibliografie}

Fortschr Röntgenstr 2020; 192: 1077-1079

Online-Publikation: 14.5.2020

DOI $10.1055 / a-1157-9537$

ISSN 1438-9029

(C) 2020. Thieme. All rights reserved.

Georg Thieme Verlag KG, Rüdigerstraße 14

70469 Stuttgart, Germany 